\title{
Electrical Energy Forecasting and Optimal Allocation of ESS in a Hybrid Wind-Diesel Power System
}

\author{
Hai Lan ${ }^{1}$, He Yin ${ }^{1}$, Shuli Wen ${ }^{1, *}$, Ying-Yi Hong ${ }^{2}$, David C. Yu ${ }^{3}$ and Lijun Zhang ${ }^{1}$ \\ 1 College of Automation, Harbin Engineering University, Harbin 150001, China; lanhai@hrbeu.edu.cn (H.L.); \\ ccy1824@hrbeu.edu.cn (H.Y.); zhangli7385@nwpu.edu.cn (L.Z.) \\ 2 Department of Electrical Engineering, Chung Yuan Christian University, Chung Li District 320, \\ Taoyuan City 32023, Taiwan; yyhong@ee.cycu.edu.tw \\ 3 Department of Electrical Engineering and Computer Science, University of Wisconsin-Milwaukee, \\ Milwaukee, WI 53211, USA; yu@uwm.edu \\ * Correspondence: wenshuli@hrbeu.edu.cn; Tel.: +86-451-82568560
}

Academic Editors: Josep M. Guerrero and Amjad Anvari-Moghaddam

Received: 4 November 2016; Accepted: 25 January 2017; Published: 14 February 2017

\begin{abstract}
Due to the increasingly serious energy crisis and environmental pollution problem, traditional fossil energy is gradually being replaced by renewable energy in recent years. However, the introduction of renewable energy into power systems will lead to large voltage fluctuations and high capital costs. To solve these problems, an energy storage system (ESS) is employed into a power system to reduce total costs and greenhouse gas emissions. Hence, this paper proposes a two-stage method based on a back-propagation neural network (BPNN) and hybrid multi-objective particle swarm optimization (HMOPSO) to determine the optimal placements and sizes of ESSs in a transmission system. Owing to the uncertainties of renewable energy, a BPNN is utilized to forecast the outputs of the wind power and load demand based on historic data in the city of Madison, USA. Furthermore, power-voltage $(P-V)$ sensitivity analysis is conducted in this paper to improve the converge speed of the proposed algorithm, and continuous wind distribution is discretized by a three-point estimation method. The Institute of Electrical and Electronic Engineers (IEEE) 30-bus system is adopted to perform case studies. The simulation results of each case clearly demonstrate the necessity for optimal storage allocation and the efficiency of the proposed method.
\end{abstract}

Keywords: renewable energy; energy storage system; hybrid multi-objective particle swarm optimization; back-propagation neural network; power-voltage sensitivity analysis; three-point estimation method

\section{Introduction}

With the rapid development of renewable energy, interest in wind power has drawn more attention, as it possesses advantages such as free energy resources, non-greenhouse gas emission, and the ability of supporting rural areas. However, a high penetration of wind power raises a problem of system instability, caused by the nature of wind uncertainty. The integration of ESS is one of the best solutions to guarantee a stable power system with distributed wind resources [1].

An optimal allocation of ESSs in power systems can reduce total costs, enhance reliability and power quality, and, by determining the best locations and sizes of ESSs, improve voltage profiles [2]. Studies $[3,4]$ show that an optimal planning of locations and sizes of ESSs in power systems can reduce a power system's costs and enhance a its reliability and power quality. A novel method has been presented in [5] for designing an energy storage system dedicated to the reduction of the uncertainty of short-term wind power forecasts up to $48 \mathrm{~h}$. Wang et al. in [6] proposed a determination methodology for optimizing the capacity of an ESS that enables a wind power generator to meet the requirements of grid integration. To improve regulation effects, the segmentation method and automatic segmentation 
method are also applied to the proposed algorithm. An improved genetic algorithm is utilized in [7] to obtain the best energy savings and voltage profile by optimizing the location and size of ultra-capacitors. In [8], Xiao et al. proposed a capacity optimization method for a hybrid energy storage system taking SOC and efficiency into account. They used the maximal cumulative capacity and SOC constraint to calculate ESS capacity. Motaleb et al. [9] performed optimal sizing for a hybrid power system with wind and energy storage sources based on stochastic modeling of historical wind speed and load demand.

Since the uncertainties of wind power will lead to large errors in the results of optimally allocating ESSs, a power forecasting method is always used to predict power demand, spot price, and outputs of renewable energy and helps decision-makers determine an ESS's capacity more accurately. A large amount of literature [10-13] related to power prediction has been developed in recent years. The authors of [14-16] utilized artificial neural network (ANN) approaches for the optimum estimation and forecasting of renewable energy consumption by considering environmental and economic factors. Physical models were set up to predict solar irradiance and the output power of photovoltaic (PV) generation, which are based on numerical weather predictions and satellite images $[17,18]$. Bacher et al. developed a statistical approach based on a data-driven formulation using historical measured data to forecast renewable energy time series [19].

In addition to the determination of ESS capacity, it is also necessary to design the placement and rated power of energy storing devices, which is a complex parameter optimization problem. A multi-objective particle swarm optimization (MOPSO) algorithm has been widely utilized to solve the nonlinear, non-differentiable, multidimensional optimization problems [20,21]. Ganguly in [22] made use of MOPSO to plan the reactive power compensation of radial distribution networks with a unified power quality conditioner. Ramadan et al. [23] adopted the MOPSO technique to find the best capacitors in distribution systems that are connected to wind energy generations.

The focus of this paper is to optimally allocate ESSs in a power system integrated with wind power taking system costs, carbon dioxide emissions, and voltage fluctuations into account. In order to mitigate the influence of the uncertainties of wind power, a back-propagation neural network technique is utilized to forecast the power gap of the load and wind power using historical data. A hybrid multi-objective particle swarm optimization algorithm, which consists of a three-point estimation method, MOPSO, and a probabilistic power flow method, is developed to optimize the placements and sizes of the ESSs. Furthermore, power-voltage sensitivity analysis is proposed in this paper to select candidate buses for the installation of the ESS with the purpose of minimizing iteration times.

The novelty of this work, distinguishing it from previous studies, is as follows: (1) the candidate buses for the ESSs' installation were selected by the power-voltage $(P-V)$ sensitivity analysis, which reduced the computational burden; (2) the capacity of the total ESS was determined by a forecasting method; (3) instead of running the Monte Carlo method, a three-point estimation method was employed to discretize the wind distribution; and (4) both the cost and greenhouse gas emissions were reduced by hybrid multi-objective particle swarm optimization.

The rest of this paper is organized as follows: Section 2 formulates the problem. Section 3 presents the method for solving it. Section 4 describes several case studies to demonstrate the proposed algorithm, and Section 5 draws conclusions.

\section{Problem Formulation}

\subsection{Electrical Energy Forecasting}

To ensure the secure and economic integration of wind turbines into a power system, accurate wind power and load demand forecasting has become critical of energy management systems [24]. In this paper, the back-propagation algorithm based on the artificial neural network is employed to fit 
the power curve of the difference between wind generation and load. The total capacity of the ESS is determined by the predicted wind power and loads.

Due to the uncertainties of renewable energy, it is difficult to predict the change in power and capacity of the ESSs. In this paper, the optimal storage capacity problem is formulated as a time series forecasting problem.

The outputs of the generation comprise two parts:

$$
\left\{\begin{array}{l}
P_{d}=P_{s}+P_{d e v}^{d} \\
P_{w}=P_{F}^{w}+P_{d e v}^{w}
\end{array}\right.
$$

Similarly, the load can be separated into two components as follows:

$$
P_{l}=P_{F}^{l}+P_{d e v}^{l}
$$

Supposing that

$$
P_{S}+P_{F}^{w}=P_{F}^{l}
$$
the ESS.

The power difference between the actual power and the forecasting power is compensated by

According to the requirement of a power system, the power balance in the hybrid wind/diesel/ESS system should be followed as Equation (4).

$$
P_{d}+P_{e}+P_{w}=P_{l}
$$

Consequently, the charging or discharging power of the ESS can be calculated by Equation (4) and the capacity of the ESS can be obtained herein, which is described by Equation (5).

$$
\begin{gathered}
P_{e}=P_{l}-P_{d}-P_{w}=\left(P_{F}^{l}+P_{d e v}^{l}\right)-\left(P_{s}+P_{d e v}^{d}\right)-\left(P_{F}^{w}+P_{d e v}^{w}\right) \\
C_{E}=E_{E}^{\max } / u_{\min }=P_{e}^{\max } \times \Delta t / u_{\min }
\end{gathered}
$$

Noted that the main effect of the ESS is to compensate for the power deviation from the forecast; as a result, the largest forecasting error is the total capacity of the ESSs.

\subsection{Optimal Allocation of ESSs}

The optimal placements and rated power of ESSs are formulated as a constrained nonlinear integer optimization problem where both the locations and sizes of the storage devices are discrete. The objective function encompasses the expected system costs, the emissions, and the voltage fluctuation under the consideration of multiple equality and inequality constraints.

\subsubsection{Objective Function}

The aims of this work are to minimize the total costs, greenhouse gas emissions, and voltage fluctuations by optimally determining the location and sizes of ESSs, while considering the uncertainties of the wind power generation. More specifically, the system costs contain the fuel cost of diesel generators, the operation cost of the WT, and the ESSs. The multi-objective functions are shown as follows:

$$
\left\{\begin{aligned}
\min f 1 & =\sum_{i=1}^{3} \text { Prob }_{i} \cdot \text { Cost }_{i} \\
\min f 2 & =\sum_{i=1}^{3} \text { Prob }_{i} \cdot \text { Emission }_{i} \\
\min f 3 & =\sum_{i=1}^{3} \text { Prob }_{i} \cdot F_{i}
\end{aligned}\right.
$$


Notice that

$$
\begin{gathered}
\operatorname{Cost}_{i}=\sum_{j=1}^{N} C\left(P_{d}\right)+C_{w}+C_{e}=\sum_{j=1}^{N}\left(a+b \cdot P_{d}+c \cdot P_{d}^{2}\right)+c^{w} \cdot P_{w}+c^{e} \cdot P_{e} \\
\text { Emission }_{i}=\sum_{j=1}^{N} E\left(P_{d}\right)=\sum_{j=1}^{N}\left(d+e \cdot P_{d}+f \cdot P_{d}^{2}\right) \\
F_{i}=\sum_{k=1}^{n}\left(\frac{V_{k}-V_{k}^{\text {spec }}}{\Delta V_{k}^{\max }}\right)
\end{gathered}
$$

where $i$ is the scenario caused by the three-point estimate of wind power; $V_{k}^{\text {spec }}$ is the expected voltage; $\Delta V_{k}^{\max }$ is the maximum of voltage deviation. The expected value of Equation (7) is to calculate the desired system cost and the emissions by optimally allocating the ESSs and by determining the outputs of all the different types of generators factoring in the wind distribution. However, the voltage will fluctuate sharply with the change in the wind power generation, so the voltage profile is improved by the third objective function of (7).

\subsubsection{Constraints}

To a hybrid wind/diesel/ESS power system, the following operational constraints should be satisfied.

1. Equality Constraints: the power balance that is related to the nonlinear power flow equations is considered in this paper, which is shown in Equation (11).

$$
\left\{\begin{array}{c}
P_{i}-V_{i} \sum_{j=1}^{N} V_{i}\left(G_{i j} \cos \delta_{i j}+B_{i j} \sin \delta_{i j}\right)=0 \\
Q_{i}-V_{i} \sum_{j=1}^{N} V_{i}\left(G_{i j} \sin \delta_{i j}-B_{i j} \cos \delta_{i j}\right)=0
\end{array}\right.
$$

2. Inequality Constraints: the inequality constraints are those associated with the bus voltage $V_{k}$, the reactive power of generation $Q_{G i}$, the tap of the transformer $T_{i}$, and the maximum charge/discharge power of the ESS.

$$
\left\{\begin{array}{l}
V_{\min } \leq V_{k} \leq V_{\max } \\
T_{\min } \leq T_{i} \leq T_{\max } \\
Q_{G \min } \leq Q_{G i} \leq Q_{G \max } \\
-n_{c} \cdot C_{E} \leq P_{e} \leq n_{d} \cdot C_{E}
\end{array}\right.
$$

where $n_{c}$ and $n_{d}$ are taken to be $3 \mathrm{C}$ in this paper.

\section{Solution Method}

\subsection{Discretizing Wind Distribution}

The optimal allocation of the ESSs is always determined in the worst case (peak load without wind power) or the historical time series of the power $[25,26]$. The goal of this paper is to obtain the sitting and sizing of the ESSs in a more convenient way. Instead of using the Monte Carlo method, a three-point estimation method is adopted to discretize the distribution of wind power. 


\subsubsection{Wind Distribution}

The probabilistic description of wind speed can be accurately presented by the Weibull distribution [27]. Due to the great applicability, Weibull distribution has been widely used to describe the probably distribution of the wind speed, which is defined as follows:

$$
f(x ; \lambda, k)=\frac{k}{\lambda}\left(\frac{x}{\lambda}\right)^{k-1} e^{-(x / \lambda)^{k}}
$$

where $k$ represents the shape parameter, and $\lambda$ is the scale parameter.

To obtain the wind power distribution, a linear approximation equation that established the relationship between the wind speed and the wind power is presented in (14):

$$
Y= \begin{cases}0 & \text { if } x \leq V_{c i} \text { or } x>V_{c o} \\ \alpha+\beta x & \text { if } V_{c i} \leq x \leq V_{n o} \\ M & \text { if } V_{n o} \leq x \leq V_{c o}\end{cases}
$$

where $M$ is the maximum power of wind turbine; $\alpha$ and $\beta$ are the linear coefficients; and $V_{c i}, V_{c o}$, and $V_{n o}$ denote the cut-in wind speed, the cut-out wind speed, and the normal wind speed, respectively.

\subsubsection{Discretizing Wind Speed Distribution}

The aim of the discretization scheme is to group the values of the continuous random variable into a three finite group.

The continuous sequence of wind speed can be discretized into three points, as shown in the following formula:

$$
y_{i}=\mu_{x}+z_{i} \cdot \sigma_{x} .
$$

Notice that

$$
\left\{\begin{array}{l}
z_{i}=\frac{\lambda_{3}}{2}+(-1)^{3-i} \sqrt{\lambda_{4}-\frac{3 \lambda_{3}^{2}}{4}}, i=1,2 \\
z_{3}=0
\end{array}\right.
$$

where $y_{i}$ is the discrete wind speed

Define

$$
\left\{\begin{aligned}
\mu_{x} & =\int_{0}^{M} x f(x ; \lambda, k) d x \\
\sigma_{x} & =\int_{0}^{M}\left(x-\mu_{x}\right)^{2} f(x ; \lambda, k) d x \\
\lambda_{i} & =\int_{0}^{M}\left(\frac{x-\mu_{x}}{\sigma_{x}}\right)^{i} f(x ; \lambda, k) d x
\end{aligned}\right.
$$

where $\mu_{x}$ is the mean value of $x ; \sigma_{x}$ is the standard deviation of $x ; \lambda_{i}$ is the $i$ th central moment of $x$.

Notice that

$$
\left\{\begin{array}{l}
P_{i}=\frac{(-1)^{3-i}}{z_{i}\left(z_{1}-z_{2}\right)}, i=1,2 \\
P_{3}=1-P_{1}-P_{2}
\end{array}\right.
$$

where $P_{i}$ is the probability corresponding to $y_{i}$.

A ten-year daily wind speed data for the city of Madison, USA, was utilized to fit the Weibull distribution. Specifically, the rated power for wind generation was $113 \mathrm{MW}$ with the total maximum load of $283 \mathrm{MW}$, and the cut-in, cut-out, and normal speed of wind turbines was $3.5 \mathrm{~m} / \mathrm{s}, 40 \mathrm{~m} / \mathrm{s}$, and $13.5 \mathrm{~m} / \mathrm{s}$, respectively. The three-point discrete distribution was calculated and is shown in Figure 1. 


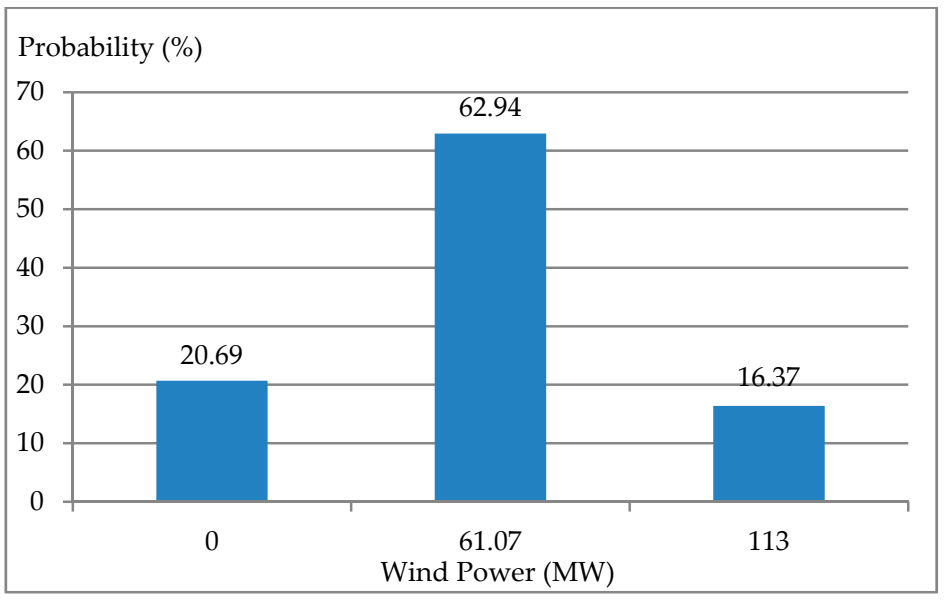

Figure 1. Three-point discrete distribution of wind power.

\subsection{BPNN Prediction}

A standard BPNN is a multilayer feed-forward neural network with error backward propagation, including an input layer, one and more hidden layers, and an output layer. Each layer is composed of a number of neurons that are connected by weights and thresholds. To solve a complicated problem, a complex neural network structure must be established by increasing the number of neurons and layers, and the structure of the network should match the problem [28]. Figure 2 illustrates a three-layer BPNN with a sigmoid function for the hidden layer and a linear function for the output layer.

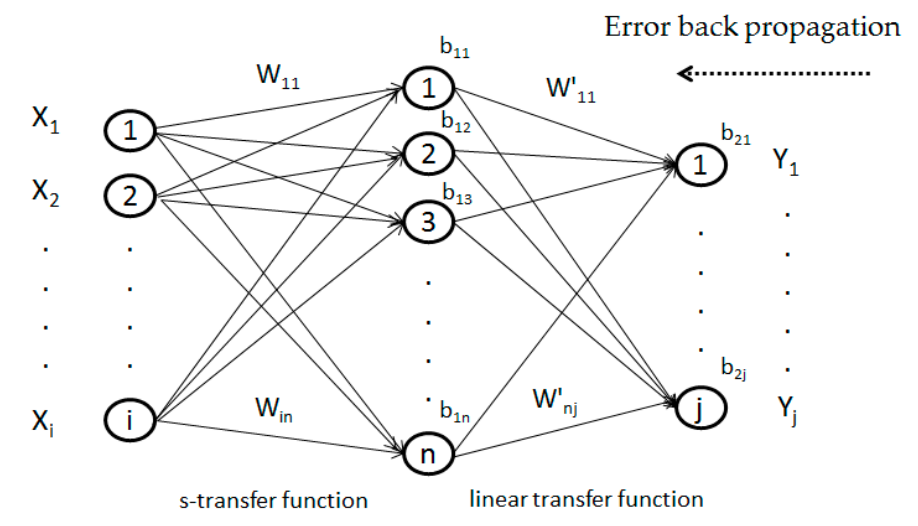

Input Layer Hidden Layer Output Layer

Figure 2. Basic structure of a back-propagation network.

During the training progress, an input pattern is given to the input layer of the network. Based on the given input pattern, the network will compute the output in the output layer. This network output is then compared with the desired output pattern. The aim of the back-propagation learning rule is to define a method of adjusting the weights of the networks. Eventually, the network will give the output that matches the desired output pattern given any input pattern in the training set.

In this paper, a dynamic artificial neural network integrated with the non-linear auto-regressive model with exogenous inputs (NARX) $[29,30]$ is employed to forecast the output power of the generation, which contains an input layer, a hidden layer with delays, and an output layer with 2,10 , and 1 neurons, respectively. 
As can be seen from Figure 3, a non-linear autoregressive with exogenous inputs dynamic neural network model establishes a relationship between the optimal scheduling of diesel generation and the actual data of wind power and the load, and is detailed in Equation (19).

$$
P_{\text {diesel }}(t)=f\left[P_{\text {diesel }}(t-1), \ldots, P_{\text {diesel }}(t-d) ; P_{\text {wind }}(t-1), \ldots, P_{\text {wind }}(t-d)\right]+\varepsilon(t), d \geq 1
$$

where $d$ is the delay-order of independent variable; $P_{\text {wind }}(t)$ is wind power at $t$ hour; $P_{\text {diesel }}(t)$ is the output power of the diesel generation power in one-hour ahead scheduling. It should be noticed that, in the NARX model, load demand is not the independent variable because it has been contained in $P_{\text {diesel }}(t)$.

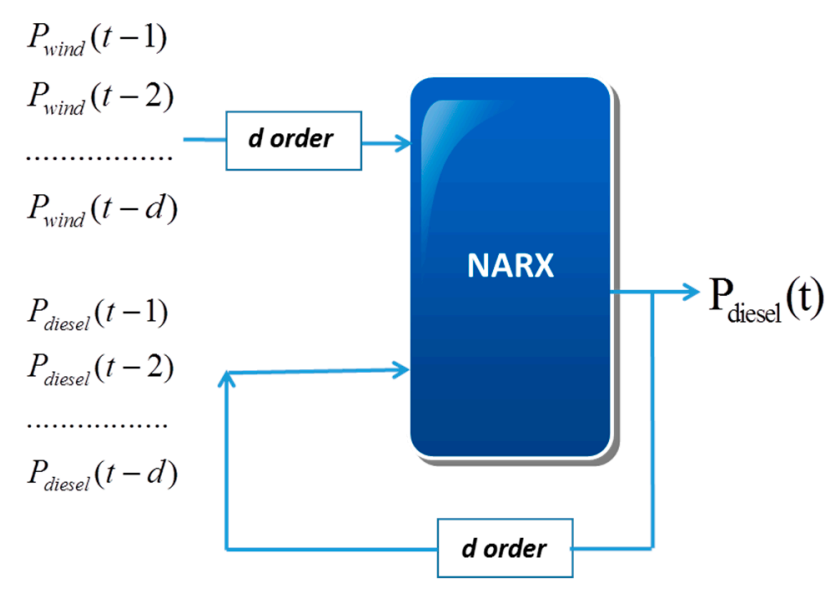

Figure 3. Non-linear auto-regressive model with exogenous inputs-back-propagation neural network (NARX-BPNN).

\subsection{HMOPSO}

Particle swarm optimization (PSO) is an intelligent optimization technique that was firstly put forward in 1995 [31-33], and the fundamental idea of the PSO algorithm is to randomly generate particles in the solution space and make each particle gradually approach the optimal solution [34,35]. In this paper, a hybrid MOPSO algorithm integrated with a non-dominated sorting genetic algorithm (NSGA-II) is programmed by MATLAB (Version R2010b, the MathWorks, Natick, MA, USA, 2010) to optimize the placements and sizes of ESSs in a hybrid wind/diesel power system. MOPSO is utilized to update the position and velocity for each particle to search the best allocation of the ESS, and NSGA-II is used to find a substantially improved spread of solutions and an improved convergence [36]. The procedure of the proposed method can be summarized as follows:

1. Forecast the difference between wind power and load demand and calculate the total capacity of the ESSs.

2. Randomly generate a population with a certain number of particles for initializing all generators' voltage, the output power, and the position and size of the ESSs. The random selections of the swarm of particles considering constraints and corresponding velocity for each particle are initialized.

3. Discretize the joint wind power distribution into a three-point distribution by the proposed estimation method, which is discussed in Section 3.1.

4. Select the candidate buses for installing ESSs via $P-V$ sensitivity analysis.

5. Through probabilistic power flow, evaluate the particles by fitness function and recall their best positions associated with the best fitness value.

6. Check and preserve the pbest (particle best) and gbest (global best); if the algorithm has not yet found the minimum cost, emissions, and voltage fluctuations, update the pbest and gbest. 
7. Duplicate the initial population to another population to form a combined population and update the position and velocity of each particle.

8. Sort the members in the new population through NSGA-II with an elitism algorithm for selecting the best solutions to renew the original population.

9. Repeat Steps 5-8 until all scenarios are considered.

\section{Results and Discussion}

\subsection{Electrical Energy Forecasting}

One-year wind power and load historical data, which are shown in Figure 4, of the hybrid system are used to predict the output power of the diesel generation.

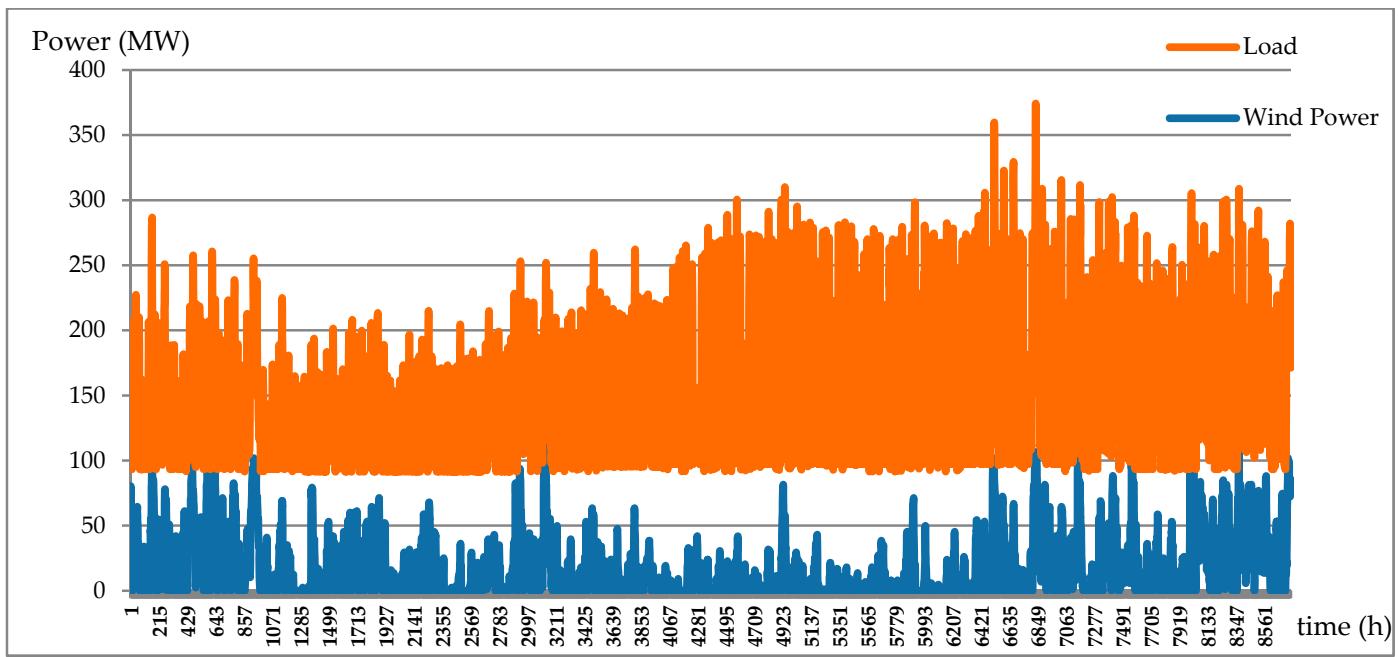

Figure 4. One-year data of wind generation and load.

In this paper, a sodium-sulfur battery is used as the energy storage unit and the slowest battery charge/discharge rate is $0.5 \mathrm{C}$. Three types of forecasting methods are studied and conducted to calculate the time series of the outputs of the generations. The total capacity of the ESSs is determined herein. The performances of the predicting methods are compared, and the results are depicted in Table 1.

Table 1. Energy storage system (ESS) capacity based on different forecasting methods.

\begin{tabular}{cccccc}
\hline Method & Network Type & Capacity (MWh) & Maximum Deviation & Mean Deviation & Variance \\
\hline No prediction & - & 1465.68 & $100 \%$ & $21.34 \%$ & 0.00686 \\
Persistence model & - & 226 & $52.09 \%$ & $8.58 \%$ & 0.009 \\
& Standard BP & 217.48 & $50.13 \%$ & $5.28 \%$ & 0.00695 \\
& Variable gradient BP & 213.56 & $49.23 \%$ & $5.30 \%$ & 0.00634 \\
Static prediction & BFGS & 205.84 & $47.45 \%$ & $5.13 \%$ & 0.00562 \\
& Conjugate gradient BP & 209.57 & $48.31 \%$ & $5.02 \%$ & 0.00533 \\
Dynamic & LM & 201.07 & $46.35 \%$ & $4.72 \%$ & 0.00484 \\
prediction & NAR BP & 56.52 & $13.03 \%$ & $0.86 \%$ & 0.00022 \\
& NARX BP & 54.4 & $12.54 \%$ & $0.89 \%$ & 0.00019 \\
\hline
\end{tabular}

It can be seen from Table 1 that, with the help of the NARX-BPNN method, the total capacity of the ESS is reduced from $1465.68 \mathrm{MWh}$ to $54.4 \mathrm{MWh}$ with a minimum variance of 0.00019 . Compared with the other prediction model, not only the maximum deviation but also the mean deviation of the dynamic prediction method achieves the lowest, which is nearly 4 times less than that of the persistence model. Figure 5 presents the performance of the NARX-BPNN method. 


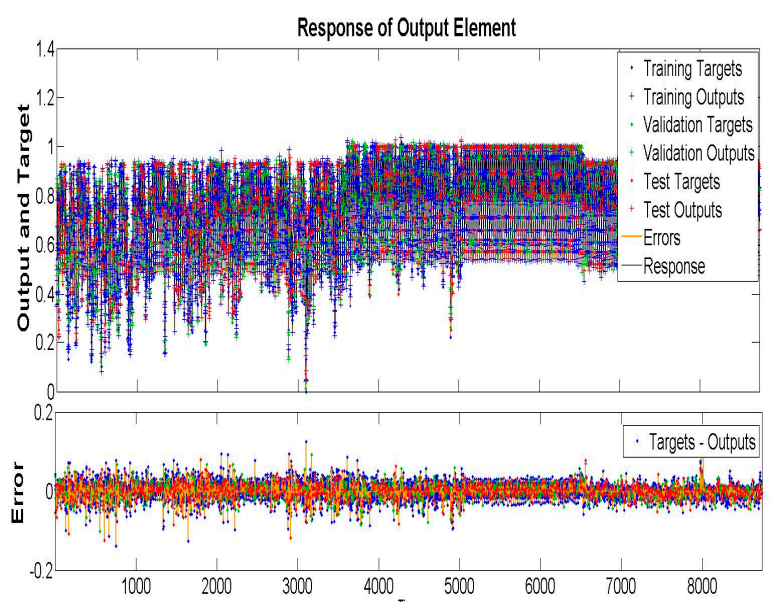

(a)

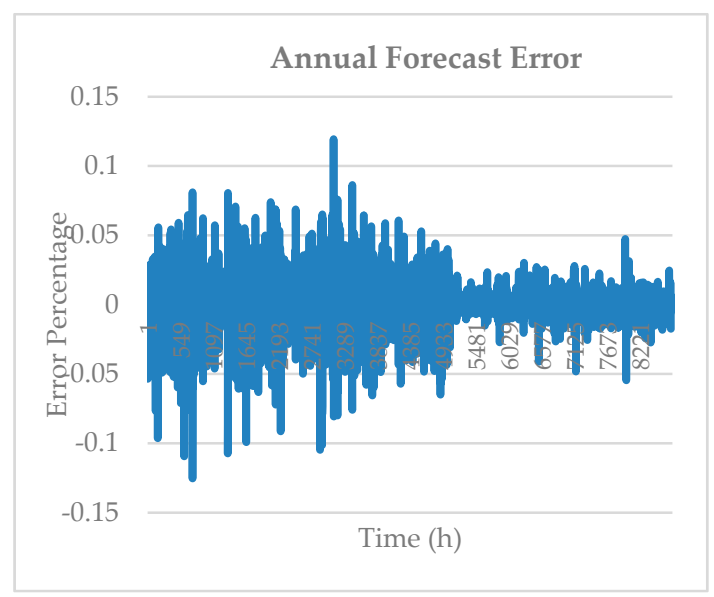

(b)

Figure 5. (a) Simulation result of NARX-BPNN; (b) Annual forecast error of NARX-BPNN.

\subsection{Sensitivity Analysis}

Power vs. voltage characteristics, known as the $P$ - $V$ curve, has been widely used as a complementary analytical tool to the dynamic study by many utilities. It depicts the loading and generating capability of each bus in the power network with respect to voltage stability. The $P-V$ curve enables system planners and operators to reduce the risks of systems accidentally entering unstable regions [37].

In the paper, in order to minimize the possible placements of energy storage systems, thereby reducing the computational complexity of the PSO algorithm, $P-V$ sensitivity analysis is conducted. The IEEE 30-bus system is selected to verify the ability performance of the proposed algorithm. The system consists of five generations and 20 loads, where Bus 1 is the slack bus, Bus 2 is installed with wind generation rated as $113 \mathrm{MW}$, Buses 5, 811 , and 13 are defined as PV nodes, and other buses are PQ nodes [38]. Wind generation is added to Bus 2 rated as $113 \mathrm{MW}$. In order to simulate the extreme actual severe operating condition and expose the weaknesses and limitations of the system, the lengths of branches from Bus 1 to Bus 3 and Bus 1 to Bus 2 are doubled.

Buses that have more variation according to the change of loading and/or generating conditions are identified as sensitive buses. By placing energy storage systems at sensitive buses, the voltage profiles not only at these sensitive buses but also at adjacent buses will be improved. Therefore, by selecting proper locations for energy storage systems, the overall system voltage profiles can be improved, and system costs and losses thus minimized.

Figure 6 depicts $P-V$ curves of generation and load areas in the IEEE 30-bus system. Buses 7, 10, and 12 in the generation area shown in Figure 6a, and Buses 25, 26, 28, the 30 in the load area shown in Figure $6 \mathrm{~b}$ are noticeably more sensitive than other buses; in other words, they are able to contribute more voltage stability than other buses when energy storage devices are installed. It is worth noticing that Buses 1, 5, 8, 11, and 13 are not considered to install ESSs, as they are either connected to the bulk grid or already installed generations. 


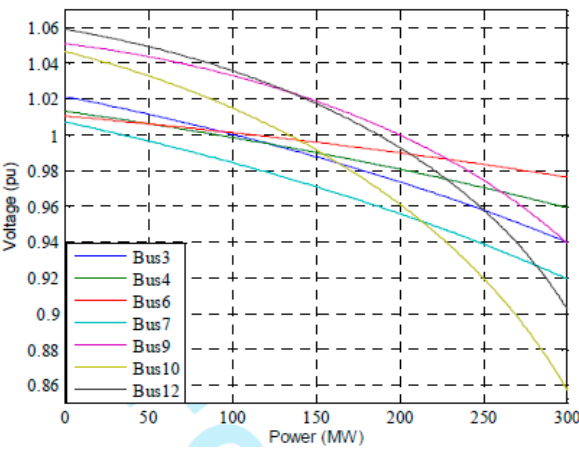

(a)

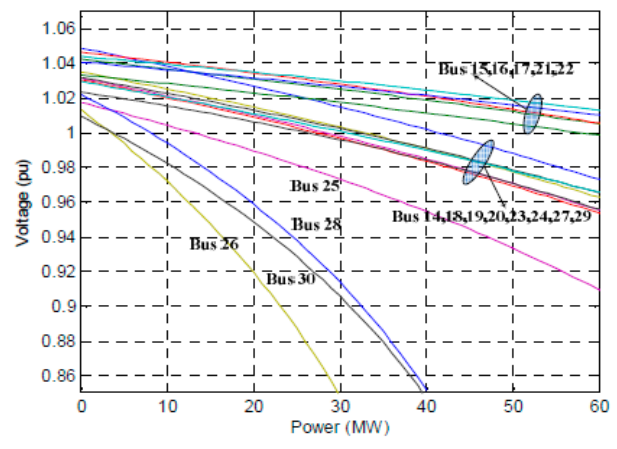

(b)

Figure 6. (a) Power-voltage $(P-V)$ curves for generation areas of the IEEE 30 bus system; (b) $P-V$ curve for loading areas of the Institute of Electrical and Electronic Engineers (IEEE) 30 bus system.

\subsection{Economic Analysis}

Considering the actual situation, the impacts of integration of the wind power and ESS into a transmission system are studied and compared in three cases in consideration of three scenarios of wind power, which are obtained from 3-point estimation method (3-PEM) to illustrate the effectiveness of the HMOPSO method. Table 2 presents the fuel cost parameters for each diesel engine, and the operation cost parameters $c^{w}, c^{e}$ are $45 \$ / \mathrm{MW}$ and $5.8 \$ / \mathrm{MW}$, respectively.

Table 2. Fuel cost parameters of diesel engines.

\begin{tabular}{cccc}
\hline Generator & $\boldsymbol{a}$ & $\boldsymbol{b}$ & $\boldsymbol{c}$ \\
\hline Gen 1 & 0 & 20 & 0.038432 \\
Gen 2 & 0 & 40 & 0.01 \\
Gen 3 & 0 & 40 & 0.01 \\
Gen 4 & 0 & 40 & 0.01 \\
Gen 5 & 0 & 40 & 0.01 \\
\hline
\end{tabular}

Case 1: A probabilistic load flow analysis for the peak load condition in the IEEE 30-bus system without ESS installation;

Case 2: A cost study with an ESS completely installed in Bus 2 where wind turbine is located;

Case 3: HMOPSO considering seven sensitive nodes as candidate locations.

Figure 7 reveals the economic performance of optimization results in three cases.

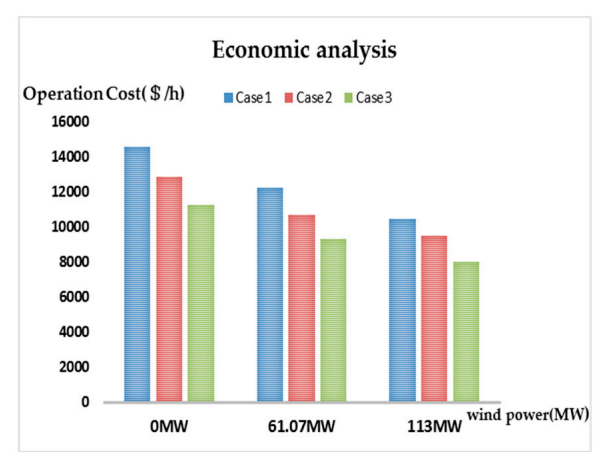

(a)

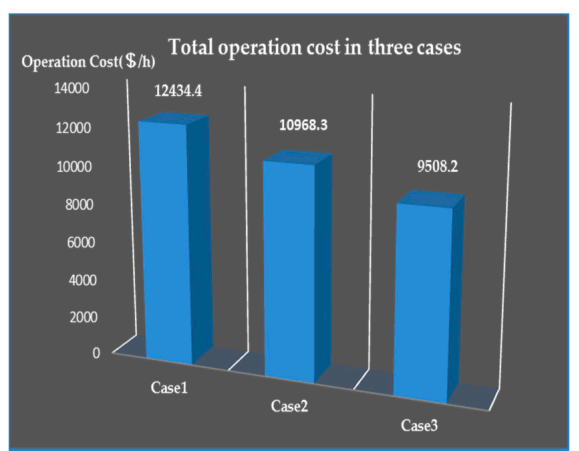

(b)

Figure 7. (a) Operation cost with consideration of wind distribution; (b) Total operation cost in three cases. 
It can be seen from above that the total operation cost is reduced with the changes of the wind power from $0 \mathrm{MW}$ to $113 \mathrm{MW}$. In Case 1, the load demand is only supplied by the diesel generation such that the system has to face a high cost of $\$ 12,434.4 / \mathrm{h}$. Even though the ESS is introduced to Case 2, the system cost is still high which implies that the distributed ESSs and optimization method is necessary. More specifically, the costs in Case 2 are $\$ 12,877, \$ 10,721$, and $\$ 9507$ with the change of the wind power from $0 \mathrm{MW}$ to $113 \mathrm{MW}$. Compared with other cases, the cost in Case 3 is the lowest with $\$ 11,270 / \mathrm{h}, \$ 9312 / \mathrm{h}$, and $\$ 8036 / \mathrm{h}$, respectively corresponding to the three different wind power outputs. If the system operate in one year $(8760 \mathrm{~h})$, the system will save $\$ 25,633,512$.

\subsection{Carbon Emission Analysis}

Table 3 presents the carbon emission parameters of diesel engines [39]. Figure 8 reveals the benefit from the renewable energy. It shows that total carbon emission decreased from 17,064 kg/h to $15,364 \mathrm{~kg} / \mathrm{h}$ with increasing wind power in Case 1 . Compared with Case 1 , the greenhouse gas emissions dropped by $5.9 \%$ every hour, which is $15,194.4 \mathrm{~kg} / \mathrm{h}$ in Case 3 . The total carbon emission is markedly decreased from $16,151.27 \mathrm{~kg} / \mathrm{h}$ to $15,194.4 \mathrm{~kg} / \mathrm{h}$ owing to the optimal allocation of ESSs. It should be noted that the emissions are $17,386 \mathrm{~kg} / \mathrm{h}$ in Case 3 when the wind power reaches the maximum. The simulation results indicated that the operating range of the diesel engine should be taken into account when optimizing the configuration of the ESS.

Table 3. Carbon emission parameters of diesel engines.

\begin{tabular}{cccc}
\hline Generator & $\boldsymbol{d}$ & $\boldsymbol{e}$ & $\boldsymbol{f}$ \\
\hline Gen 1 & 22.983 & -0.9 & 0.0126 \\
Gen 2 & 25.505 & -0.01 & 0.027 \\
Gen 3 & 24.900 & -0.005 & 0.0291 \\
Gen 4 & 24.700 & -0.004 & 0.0290 \\
Gen 5 & 25.300 & -0.0055 & 0.0271 \\
\hline
\end{tabular}

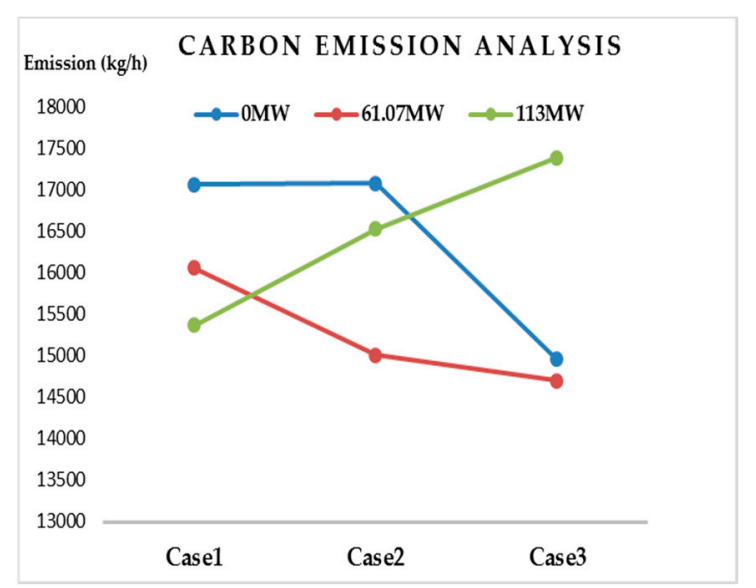

(a)

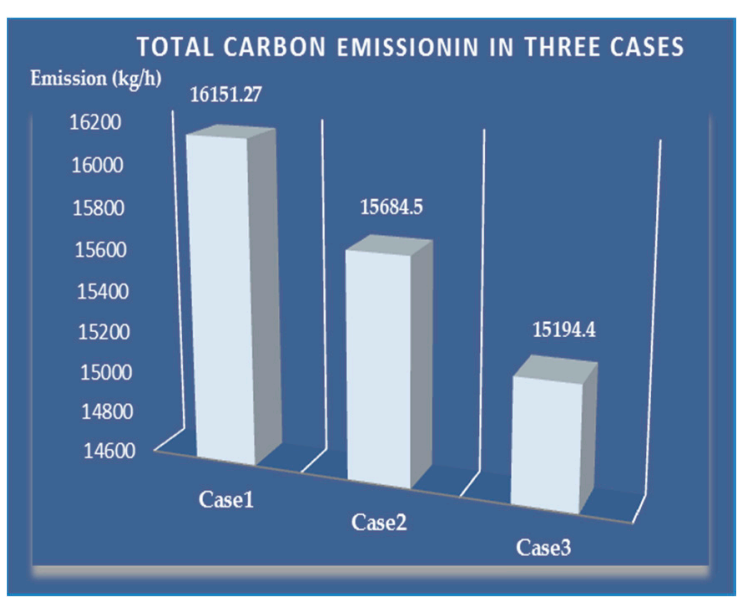

(b)

Figure 8. (a) Carbon emission considering wind distribution; (b) Total carbon emission.

As seen from the above analysis, the operation cost and carbon emission are greatly reduced by the distributed ESS configuration. Meanwhile, the real power loss of the system declines sharply from 26.4 MW to 10.7 MW. Eventually, Buses 12, 25, and 26 are found to be the best places to install ESSs with capacities of $30.7 \mathrm{MW}, 18 \mathrm{MW}$, and $39.46 \mathrm{MW}$, respectively, which are demonstrated in Table 4. 
Table 4. Optimal allocation of energy storage systems (ESSs).

\begin{tabular}{cccc}
\hline ESS & BUS 12 & BUS 25 & BUS 26 \\
\hline Rated power (MW) & 30.70 & 18.00 & 39.46 \\
Capacity (MWh) & 23.46 & 6.64 & 24.30 \\
\hline
\end{tabular}

\section{Conclusions}

With the rapid development of renewable energy, it has become important to forecast electric energy when optimally planning a stable and economic power system. In this paper, a two-stage hybrid MOPSO that integrates with a back-propagation neural network is proposed to optimize the allocation of ESSs in order to reduce total cost and emissions. Furthermore, wind power distribution is discretized by a three-point estimation method, and the $P-V$ curve is explored to select candidate buses for the installation of the ESSs. The simulation results show that (i) the dynamic prediction method is more suitable for forecasting wind power and load demand, which has the lowest number of errors; (ii) with the help of $P-V$ sensitivity analysis, the proposed HMOPSO is able to search for the best placement and size for ESSs at a fast speed as well as minimize the total operation cost and improve voltage profiles; (iii) different from conventional analysis, the distributed ESSs can achieve minimum costs and greenhouse gas emissions for a wind power integrated system. In a future study, the air density and ambient temperature will be taken into account to improve the forecasting method.

Acknowledgments: This work was supported by the Natural Science Foundation of China under Grant No. 61174047 and by Fundamental Research Funds for the Central Universities under Grant No. HEUCFZ1305.

Author Contributions: All authors contributed to this collaborative work. Hai Lan and He Yin performed the research and discussed the results. Shuli Wen designed the optimal algorithm. Ying-Yi Hong, David C. Yu, and Lijun Zhang suggested the research idea and contributed to the writing and revision of the paper. All authors approved the manuscript.

Conflicts of Interest: The authors declare no conflict of interest.

\section{Nomenclature}

$\begin{array}{ll}\text { Acronyms } & \\ \text { ANN } & \text { Artificial Neural Network } \\ \text { BFGS } & \text { Quasi-Newton } \\ \text { BPNN } & \text { Back-propagation Neural Network } \\ \text { ESS } & \text { Energy Storage System } \\ \text { HMOPSO } & \text { Hybrid Multi-objective PSO } \\ \text { LM } & \text { Levenberg Marquard } \\ \text { MOPSO } & \text { Multi-objective PSO } \\ \text { NAR } & \text { Non-linear Auto-regressive } \\ \text { NARX } & \text { Non-linear Auto-regressive model with exogenous inputs } \\ \text { NARX-BPNN } & \text { Non-linear Auto-regressive model with exogenous inputs-back-propagation neural network } \\ \text { O\&M } & \text { Operation and Management } \\ \text { PEM } & \text { Point Estimation Method } \\ \text { PSO } & \text { Particle Swarm Optimization } \\ \text { PV } & \text { Photovoltaic } \\ \text { SOC } & \text { State of Charge } \\ \text { WT } & \text { Wind Turbine }\end{array}$




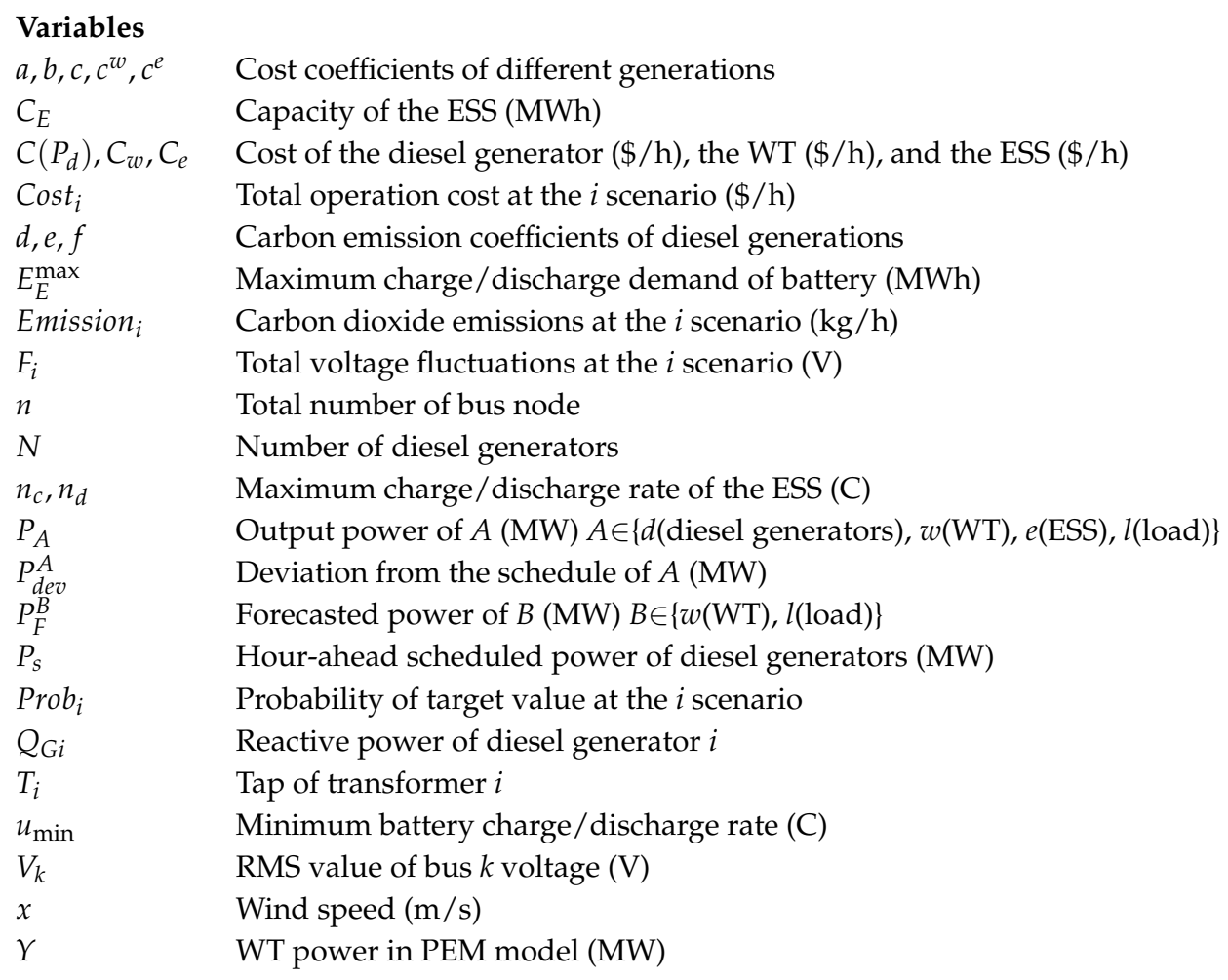

\section{References}

1. Perez, M.J.R.; Fthenakis, V.M. On the spatial decorrelation of stochastic solar resource variability at long timescales. Sol. Energy 2015, 117, 46-58. [CrossRef]

2. Ogunjuyigbe, A.S.O.; Ayodele, T.R.; Akinola, O.A. Optimal allocation and sizing of PV/Wind/Split-diesel/ Battery hybrid energy system for minimizing life cycle cost, carbon emission and dump energy of remote residential building. Appl. Energy 2016, 171, 153-171. [CrossRef]

3. Chen, C.; Duan, S.; Cai, T. Optimal allocation and economic analysis of energy storage system in microgrids. IEEE Trans. Power Electron. 2011, 26, 2762-2773. [CrossRef]

4. ElNozahy, M.S.; Tarek, K.; Abdel-Galil; Salama, M.M.A. Probabilistic ESS sizing and scheduling for improved integration of PHEVs and PV systems in residential distribution systems. Electr. Power Syst. Res. 2015, 125, 55-66. [CrossRef]

5. Bludszuweit, H.; Dominguez, N.J.A. A probabilistic method for energy storage sizing based on wind power forecast uncertainty. IEEE Trans. Power Syst. 2011, 26, 1651-1658. [CrossRef]

6. Wang, W.; Mao, C.; Lu, J. An energy storage system sizing method for wind power integration. Energies 2013, 6, 3392-3404. [CrossRef]

7. Wang, B.; Yang, Z.P.; Lin, F. An improved genetic algorithm for optimal stationary energy storage system locating and sizing. Energies 2014, 7, 6434-6458. [CrossRef]

8. Xiao, J.; Liang, H.; Yu, J.; Zhang, P.; Wang, X.; Yuan, S. A capacity optimization method for hybrid energy storage system considering SOC and efficiency. In Proceedings of the Renewable Power Generation Conference (RPG 2013), 2nd IET, Beijing, China, 9-11 September 2013; pp. 1-4.

9. Motaleb, A.M.A.E.; Bekdache, S.K.; Barrios, L.A. Optimal sizing for a hybrid power system with wind/energy storage based in stochastic environment. Renew. Sustain. Energy Rev. 2016, 59, 1149-1158. [CrossRef]

10. Antonanzas, J.; Osorio, N.; Escobar, R.; Urraca, R.; Martinez-de-Pison, F.J.; Antonanzas-Torres, F. Review of photovoltaic power forecasting. Sol. Energy 2016, 136, 78-111. [CrossRef]

11. Yan, J.; Liu, Y.; Han, S.; Wang, Y.; Feng, S. Reviews on uncertainty analysis of wind power forecasting. Renew. Sustain. Energy Rev. 2015, 52, 1322-1330. [CrossRef]

12. Hong, T.; Fan, S. Probabilistic electric load forecasting: A tutorial review. Int. J. Forecast. 2016, 32, 914-938. [CrossRef] 
13. Claudio, M.; Ignacio, J.R.R.; Alfredo, F.J.L. Short-term forecasting model for aggregated regional hydropower generation. Energy Convers. Manag. 2014, 88, 231-238.

14. Okumus, I.; Dinler, A. Current status of wind energy forecasting and a hybrid method for hourly predictions. Energy Convers. Manag. 2016, 123, 362-371. [CrossRef]

15. Mazorra, A.L.; Pereira, B.; Lauret, P. Combining solar irradiance measurements, satellite-derived data and a numerical weather prediction model to improve intra-day solar forecasting. Renew. Energy 2016, 97, 599-610.

16. Fernando, A.O.P.; Jesús, F.B.; Juan, F.G.F. Failure mode prediction and energy forecasting of PV plants to assist dynamic maintenance tasks by ANN based models. Renew. Energy 2015, 81, 227-238.

17. Giorgi, M.G.D.; Congedo, P.M.; Malvoni, M. Photovoltaic power forecasting using statistical methods: Impact of weather data. Meas. Technol. 2014, 8, 90-97. [CrossRef]

18. Alonso-Montesinos, J.; Batlles, F.J.; Portillo, C. Solar irradiance forecasting at one-minute intervals for different sky conditions using sky camera images. Energy Convers. Manag. 2015, 105, 1166-1177. [CrossRef]

19. Bacher, P.; Madsen, H.; Nielsen, H.A. Online short-term solar power forecasting. Sol. Energy 2009, 83, 1772-1783. [CrossRef]

20. Zidar, M.; Georgilakis, P.S.; Hatziargyriou, N.D. Review of energy storage allocation in power distribution networks: Applications, methods and future research. IET Gener. Transm. Distrib. 2016, 10, 645-652. [CrossRef]

21. Suchitra, D.; Jegatheesan, R.; Deepika, T.J. Optimal design of hybrid power generation system and its integration in the distribution network. Int. J. Electr. Power Energy Syst. 2016, 82, 136-149. [CrossRef]

22. Ganguly, S. Multi-objective planning for reactive power compensation of radial distribution networks with unified power quality conditioner allocation using particle swarm optimization. IEEE Trans. Power Syst. 2014, 29, 1801-1810. [CrossRef]

23. Ramadan, H.S.; Bendary, A.F.; Nagy, S. Particle swarm optimization algorithm for capacitor allocation problem in distribution systems with wind turbine generators. Int. J. Electr. Power Energy Syst. 2017, 84, 143-152. [CrossRef]

24. Muhammad, K.; Abdollah, A.; Andrey, V.S.; Vassilios, G.A. Minimizing the energy cost for microgrids integrated with renewable energy resources and conventional generation using controlled battery energy storage. Renew. Energy 2016, 97, 646-655.

25. Du, Y. Optimal allocation of energy storage system in distribution systems. Procedia Eng. 2011, 15, 346-351.

26. Lan, H.; Dai, J.; Wen, S. Optimal tilt angle of photovoltaic arrays and economic allocation of energy storage system on large oil tanker ship. Energies 2015, 8, 11515-11530. [CrossRef]

27. Fichaux, N.; Poglio, T.; Ranchin, T. Mapping offshore wind resources: Synergetic potential of SAR and scatterometer data. IEEE J. Ocean. Eng. 2005, 30, 516-525. [CrossRef]

28. Wang, S.; Zhang, N.; Wu, L.; Wang, Y. Wind speed forecasting based on the hybrid ensemble empirical mode decomposition and GA-BP neural network method. Renew. Energy 2016, 94, 629-636. [CrossRef]

29. Haddad, S.; Benghanem, M.; Mellit, A.; Daffallah, K.O. ANNs-based modeling and prediction of hourly flow rate of a photovoltaic water pumping system: Experimental validation. Renew. Sustain. Energy Rev. 2015, 43, 635-643. [CrossRef]

30. Hussain, S.; Al-Alili, A. A new approach for model validation in solar radiation using wavelet, phase and frequency coherence analysis. Appl. Energy 2016, 164, 639-649. [CrossRef]

31. Saleh, F.; Hossein, R.; Alireza, H.; Hossein, I. A novel PSO (Particle Swarm Optimization)-based approach for optimal schedule of refrigerators using experimental models. Energy 2016, 107, 707-715.

32. Kennedy, J.; Eberhart, R. Particle swarm optimization. IEEE Int. Conf. Neural Netw. 1995, 4, $1942-1948$.

33. Eberhart, R.; Kennedy, J. A new optimizer using particle swarm theory. In Proceedings of the International Symposium on MICRO Machine and Human Science, Ann Arbor, MI, USA, 4-6 October 1995; pp. $39-43$.

34. Robert, J.H.; Nacer, K.M.; Aziz, N. Optimization of hybrid renewable energy systems (HRES) using PSO for cost reduction. Energy Procedia 2013, 42, 318-327.

35. Clerc, M.; Kennedy, J. The particle swarm-explosion, stability, and convergence in a multidimensional complex space. IEEE Trans. Evol. Comput. 2002, 6, 58-73. [CrossRef]

36. Deb, K.; Kalyanmoy, D. Multi-Objective Optimization Using Evolutionary Algorithms; John Wiley \& Sons: Hoboken, NJ, USA, 2001.

37. Mohn, F.W.; Souza, A.C.Z. Tracing PV and QV curves with the help of a CRIC continuation method. IEEE Trans. Power Syst. 2006, 21, 1115-1122. [CrossRef] 
38. 30 Bus Power Flow Test Case. Available online: http://www.ee.washington.edu/research/pstca/pf30/pg tca30bus.htm (accessed on 1 August 1993).

39. Krishnamurthy, S.; Tzoneva, R. Investigation on the impact of the penalty factors over solution of the dispatch optimization problem. In Proceedings of the 2013 IEEE International Conference on Industrial Technology (ICIT), Cape Town, South Africa, 25-28 February 2013; pp. 851-860.

(C) 2017 by the authors; licensee MDPI, Basel, Switzerland. This article is an open access article distributed under the terms and conditions of the Creative Commons Attribution (CC BY) license (http:/ / creativecommons.org/licenses/by/4.0/). 